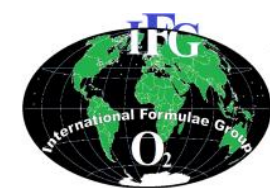

Available online at http://www.ifgdg.org

Int. J. Biol. Chem. Sci. 14(2): 539-554, February 2020

ISSN 1997-342X (Online), ISSN 1991-8631 (Print)

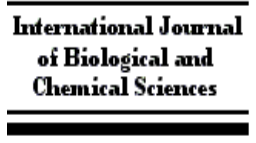

International Journal

Chemical Sciences

Original Paper http://ajol.info/index.php/ijbcs http://indexmedicus.afro.who.int

\title{
La mauvaise pratique phytosanitaire, principale source de contamination du chou au Sénégal
}

\author{
Papa Sam GUEYE ${ }^{1 *}$, Babacar LABOU ${ }^{2}$, Mamadou DIATTE ${ }^{2}$ et Karamoko DIARRA ${ }^{2}$ \\ ${ }^{1}$ Centre Régional de Recherches en Écotoxicologie et Sécurité Environnementale, Fondation CERES-Locustox. \\ Km 15, Route de Rufisque à côté de la DPV. BP. 11705, CP.13000 Dakar Peytavin Sénégal. \\ ${ }^{2} U C A D$, Département de Biologie Animale, Labo 2PIA, Dakar, Sénégal. \\ *Auteur correspondant; E-mail: psamgueye@hotmail.com ; Tel : +221775631163.
}

\section{REMERCIEMENTS}

Ce travail a été soutenu financièrement par le Centre Régional de Recherches en Ecotoxicologie et Sécurité Environnementale (CERES-Locustox).

\section{RESUME}

L'utilisation des pesticides dans l'agriculture engendre des résidus toxiques non négligeables sur les produits agricoles et sur les matrices environnementales. L'emploi frauduleux des pesticides non autorisés, le surdosage des produits actifs et leur utilisation abusive par les producteurs impactent sérieusement la santé des populations. L'objectif de cette étude est d'identifier les sources de contamination du chou à travers l'évaluation du niveau de contamination des matrices. Les échantillons de sols et d'eaux ont été prélevés conformément aux Directives de la FAO tandis que ceux du chou l'ont été en respectant la norme du Codex Alimentarius. Les résultats montrent que sur les 30 échantillons d'eau prélevés dans les puits, une seule contamination a été notée avec la Lambda-cyhalothrine à 3,93 $\mu \mathrm{g} / \mathrm{l}$. L'analyse du sol montre que 43,33\% des échantillons ont été contaminés par les pesticides. L'analyse du chou montre que $63,3 \%$ des échantillons sont contaminés. La trifluraline constitue la substance active la plus fréquente, suivie du diméthoate et de l'heptachlore (POP). Ces produits ont été retrouvés à des seuils dépassant la LMR du Codex Alimentarius. L'eau et le sol ne sont pas des sources de contamination du chou. La contamination du chou est liée à l'utilisation des pesticides par les producteurs. En effet, les produits qui contaminent le chou n'ont pas été observés dans les matrices environnementales eau et sol. Le chou, très consommé au Sénégal est un légume fortement pollué qui devrait faire l'objet d'un suivi régulier. Cette étude constitue un outil d'aide à la décision indispensable pour une meilleure gestion des pesticides.

(C) 2020 International Formulae Group. All rights reserved.

Mots clés : Chou, sol, eau, pesticides, POP, Afrique.

\section{Poor phytosanitary practice, the main source of cabbage contamination in Senegal}

\section{ABSTRACT}

The use of pesticides in agriculture generates significant toxic residues on agricultural products and on environmental matrices. The fraudulent use of unauthorized pesticides, overdose active products and their abusive 
use by producers seriously impact the health of populations. The objective of this study is to identify cabbage contamination sources through the evaluation of the level of matrices contamination. The soil and water samples were collected in accordance with FAO guidelines while those of the cabbage were respecting the Codex Alimentarius standard. The results show that out of 30 water samples taken from the wells, only one contamination was noted with Lambda-cyhalothrin at $3.93 \mu \mathrm{g} / \mathrm{l}$. Soil analysis shows that $43.33 \%$ of samples were contaminated by pesticides. The cabbage analysis shows that $63.3 \%$ of the samples were contaminated. The trifluralin is the most common active substance, followed by the dimethoate and the heptachlor (POPs). These products have been found at levels exceeding the Codex Alimentarius MRL. Water and soil are not sources of cabbage contamination. Cabbage contamination is related to the use of pesticides by farmers. In fact, the products that contaminate cabbage have not been observed in environmental matrices (water and soil). Cabbage, which is widely consumed in Senegal, is a highly polluted vegetable that should be monitored regularly. This study is an indispensable decision support tool for better pesticide management.

(C) 2020 International Formulae Group. All rights reserved.

Keywords: Cabbage, soil, water, pesticides, POPs, Africa.

\section{INTRODUCTION}

Au Sénégal, l'agriculture périurbaine pratiquée dans la zone des Niayes occupe une place importante dans l'approvisionnement des populations en produits agricoles (Ngom et al., 2012).

Le chou représente $7,7 \%$ de la production légumière annuelle dans la zone, soit 55000 tonnes (ANDS, 2013). Cette culture est très sensible aux attaques de plusieurs insectes et pathogènes (Sow et al., 2013 ; Labou et al., 2016 ; Labou et al., 2017). Différentes matières actives sont utilisées seules ou en mélange pour contrôler les ravageurs du chou (Sow et al., 2013). Les pesticides utilisés, en quantités excessives ou inadaptées, parmi lesquels dominent les insecticides à large spectre, sont à l'origine de la contamination des produits maraichers et plus particulièrement du chou (Sow et Diarra, 2013 ; Tendeng et al., 2017). Les populations sont susceptibles d'être exposées à ces substances chimiques via leurs résidus dans les fruits et légumes consommés quotidiennement (Kanda et al. 2009 ; Ngom et al., 2012 ; Gouda et al., 2018). Le risque est d'autant plus inquiétant que l'endosulfan ait été détecté dans les produits agricoles (Deguine et al., 2008; Ngom et al., 2012 ; Ngom et al., 2013).

Cependant, l'origine de la contamination du chou reste inconnue. Elle pourrait être liée aux pratiques culturales ou à la contamination par les matrices environnementales (eau et sol) (Roche et al., 2005). Cette étude vise à identifier les sources de contamination du chou. Ceci à travers l'évaluation du niveau de contamination des matrices environnementales et du chou dans la zone des Niayes.

\section{MATERIEL ET METHODES \\ Présentation du site et choix des Parcelles d'étude}

Ce travail a été effectué dans la zone des Niayes. Une zone qui englobe les quatre régions bordant la frange maritime Nord du Pays que sont: Dakar, Thiès, Louga et Saint Louis (Figure 1). C'est une bande de terre qui s'étire sur une longueur de $180 \mathrm{~km}$ et d'une largeur variant de 5 à $30 \mathrm{~km}$ à l'intérieur des terres. Elle est globalement limitée dans sa partie intérieure par la route nationale Dakar-Saint Louis. La zone des Niayes est caractérisée par des conditions pédoclimatiques (climat, pédologie, hydrogéologie) favorables. Elles constituent le support des activités agropastorales telles que l'horticulture, l'aviculture et la production laitière. Les Niayes sont une terminologie régionale qui désigne une série de dépressions plus ou moins inondées de manière permanente ou semi-permanente délimitée par un ensemble de dunes externes, vives ou semi-fixées. Les Niayes de Dakar sont caractérisés par une nappe d'eau souterraine peu profonde $(0,5$ à $5 \mathrm{~m}$ de profondeur) et affleurant qui repose sur une 
nappe d'eau salée. La zone des Niayes est un écosystème favorable à l'épanouissement de beaucoup d'espèces végétales. Cependant, elle est très vulnérable de par la position superficielle de sa nappe mais aussi à cause de la perméabilité de ses sols. Dans ce contexte, l'épandage des eaux usées brutes pour l'irrigation, l'utilisation des fumiers organiques pour l'amendement des champs et de produits phytosanitaires peuvent provoquer la contamination chimique et microbiologique de la nappe d'eau souterraine, des légumes produits et par conséquent exposer les populations riveraines à des risques sanitaires.

\section{Échantillonnage}

L'échantillonnage a été effectué sur un réseau de 30 parcelles réparties le long de la zone des Niayes de Dakar à Saint-Louis. Chaque parcelle a été géo référencée et suivie sur une période de 3 mois. Le prélèvement des échantillons de sol a été réalisé selon le guide de l'Organisation des Nations unies pour l'alimentation et l'agriculture (FAO, 2004). Les échantillons de sol ont été prélevés selon la norme ISO $10381: 2005$. Des échantillons d'eau ont été prélevés dans les puits et les "céanes" utilisés comme eau d'irrigation pour la parcelle de choux conformément à la norme internationale ISO 5667-6 : 2014. Pour le chou, les prélèvements ont été effectués conformément aux Directives générales sur l'échantillonnage du Codex Alimentarius (Commission du Codex Alimentarius, 2004).

Le prélèvement des échantillons a été effectué de manière aléatoire sur 5 pieds de chou. Les 5 échantillons élémentaires prélevés ont été ensuite emballés individuellement dans un sachet en plastique.

Vingt échantillons de sol ont été prélevés sur deux diagonales de la parcelle. Un kilogramme d'échantillon final composite a été obtenu après homogénéisation des 20 échantillons primaires, emballé dans un papier aluminium et sécurisée dans un sachet en plastique.

Des échantillons d'eau de 2,5 litres conditionnés dans des bouteilles en verre ambré ont été obtenus des puits et des "céanes" (Nappe phréatique) situés dans les parcelles d'étude.

Pour chaque parcelle les trois matrices ont été échantillonnés simultanément.

L'ensemble du matériel collecté (chou, sol et eau) a été étiqueté et acheminé au laboratoire de Chimie Environnementale de CERES-Locustox pour l'analyse de leur teneur en résidus de pesticides.

\section{Analyse des résidus de pesticides}

Les matières actives recherchées dans cette analyse de résidus ont porté sur une vingtaine de molécules habituellement détectées dans l'eau et les produits maraîchers. Il s'agit principalement de la trifluraline, du chlorpyrifos, du malathion, du dicofol, du profenophos, de la lambda-cyhalothrine, de la fenvalérate, de la cyperméthrine, de l'esfenvalérate, de la deltaméthrine, de la bifenthrine, du lindane, de l'heptachlore, de l'aldrine, du parathion, de la dieldrine, de l'endrine, de la DDT et du diméthoate.

L'analyse de la teneur en résidus de pesticides (Organochlorés, Organophosphorés et Pyréthrinoïdes) dans les échantillons de chou, d'eau et de sol a été réalisée en deux phases. Une phase d'extraction et purification et une phase de détection et quantification.

Cette analyse a été faite avec le respect des exigences de la norme internationale ISO/CEI 17025. Pour les légumes, la méthode d'analyse polyvalente de détermination des résidus des pesticides par CG/MS avec extraction/purification avec de l'acétonitrile et nettoyage par SPE dispersés (norme NF EN 15662 Mai 2018) a été utilisée. Pour l'eau la norme ISO 6468 : 1996 pour l'extraction et la purification des échantillons d'eau avec leur lecture par GC/ $\mu \mathrm{ECD}$ et leur quantification par utilisation de solutions étalons a été employée. Les échantillons de sol ont été analysés selon la norme ISO 10382 : 2002 pour l'extraction et la purification avec lecture par $\mathrm{GC} / \mu \mathrm{ECD}$ et quantification par utilisation de solutions étalons. 
Analyse de la qualité sanitaire et de la contamination des matrices environnementales

L'analyse de la qualité sanitaire et de la contamination des matrices environnementales (eau et sol) est réalisée sur la base des résultats d'analyse de résidus de pesticides dans les échantillons d'eau, de sol et de chou pommé provenant des trente (30) parcelles du dispositif. La démarche a consisté à confronter les résultats obtenus sur la teneur en résidus de pesticides aux normes de l'Organisation mondiale de la santé (OMS) pour l'eau, à la Limite de Détermination ou Limite of quantification (LOQ) de la méthode pour le sol, l'eau et le chou pommé ou aux Limites Maximales de Résidus (LMR) de pesticides du Codex Alimentarius (CA) pour les échantillons de chou pommé. Les normes de l'OMS sur l'eau sont issues des Directives de qualité pour l'eau de boisson (OMS, 2017)

Alors les cas de figures suivants peuvent se présenter :

1. La teneur en résidus de pesticides (TRP) trouvée dans l'eau, le sol ou le chou < LOQ de la méthode : le pesticide recherché n'est pas présent à cette limite de détermination. L'eau, le sol ou le chou pommé n'est pas contaminé par le pesticide recherché : le sol n'est pas contaminé ou l'eau ou le chou est de bonne qualité sanitaire ;

2. La teneur en résidus de pesticides (TRP) trouvée dans l'eau, le sol ou le chou > LOQ de la méthode : le pesticide recherché est pas présent. L'eau, le sol ou le chou pommé est contaminé par le pesticide recherché. Pour l'eau et le chou pommé :

i. $\quad$ La TRP $\leq$ LMR du CA ou à la norme OMS d'eau : le pesticide recherché est présent et que l'eau ou le chou concerné est contaminé par le pesticide recherché et malgré cela sa qualité sanitaire n'est pas mauvaise ;

ii. La TRP > LMR du CA ou à la norme OMS d'eau : le pesticide recherché est présent et que l'eau ou le chou pommé concerné est contaminé par le pesticide recherché et sa qualité sanitaire n'est pas bonne et présente un risque sanitaire certain.

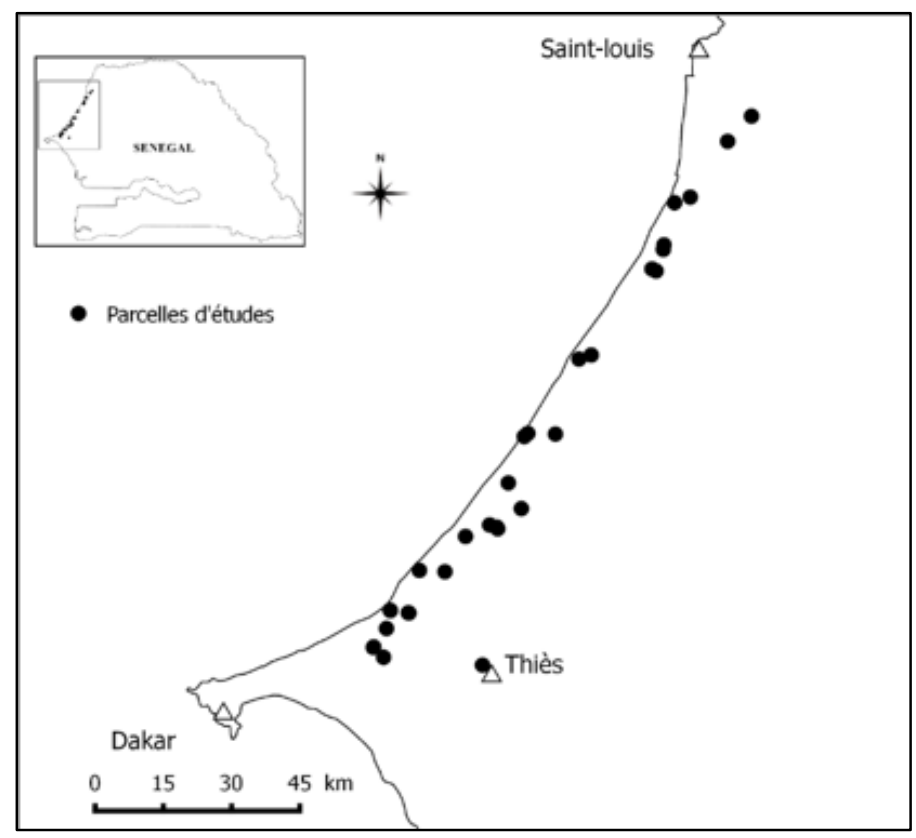

Figure 1 : Distribution spatiale des parcelles d'études dans la zone des Niayes. N=30 parcelles. 


\section{RESULTATS}

Contamination des eaux de puits et de "céanes" par les pesticides

Les résultats obtenus au niveau de la contamination de l'eau par les pesticides, en comparaison avec la valeur seuil de l'Organisation Mondiale de la Santé (OMS) montrent que sur les 30 échantillons d'eau prélevés dans les puits et "céanes", une seule contamination a été notée dans la zone centre sur les trente (30) parcelles du dispositif, soit un pourcentage de 3,33\% (Figures 2).

La matière active retrouvée est la Lambdacyhalothrine avec une teneur de 3,93 $\mu \mathrm{g} / \mathrm{l}$ soit 40 fois supérieure à la norme (Tableau 1).

\section{Contamination des sols par les pesticides}

L'analyse des échantillons de sol montre que sur les 30 échantillons, 13 ont été contaminés soit $43,33 \%$. Les parcelles les plus contaminées sont localisées dans les zones centre et sud des Niayes (Figure 2). Le DDT
(20\%), la lambda-cyhalothrine (16,7\%), l'endrine et le chlorpyrifos ( $13,3 \%$ chacun) sont les matières actives les plus fréquentes. Le profénofos, le malathion, la cyperméthrine et le lindane sont les moins fréquents avec un pourcentage de 3,3\% (Figure 3). L'endrine (613 $\mu \mathrm{g} / \mathrm{kg})$ et la Lambda-cyhalothrine $(316 \mu \mathrm{g} / \mathrm{kg})$ ont les teneurs les plus élevées (Tableau 2).

\section{Contamination du chou par les pesticides}

Les résidus de pesticides dans le chou ont été détectés dans 19 prélèvements sur les 30 , soit un pourcentage de 63,3\%. Ces contaminations sont plus élevées dans la zone centre des Niayes (Figure 2). La trifluraline $(43,3 \%)$, le diméthoate $(33,3 \%)$, l'heptachlore $(26 \%)$ et le malathion $(13,3 \%)$ sont les matières actives les plus fréquentes sur le chou (Figure 3 ) avec des teneurs respectives de 0,05 à 1,48 $\mathrm{mg} / \mathrm{kg}, 0,99-4,02 \mathrm{mg} / \mathrm{kg}, 0,01-0,33 \mathrm{mg} / \mathrm{kg}$ et 0,30-0,43 mg/kg (Tableau 3).

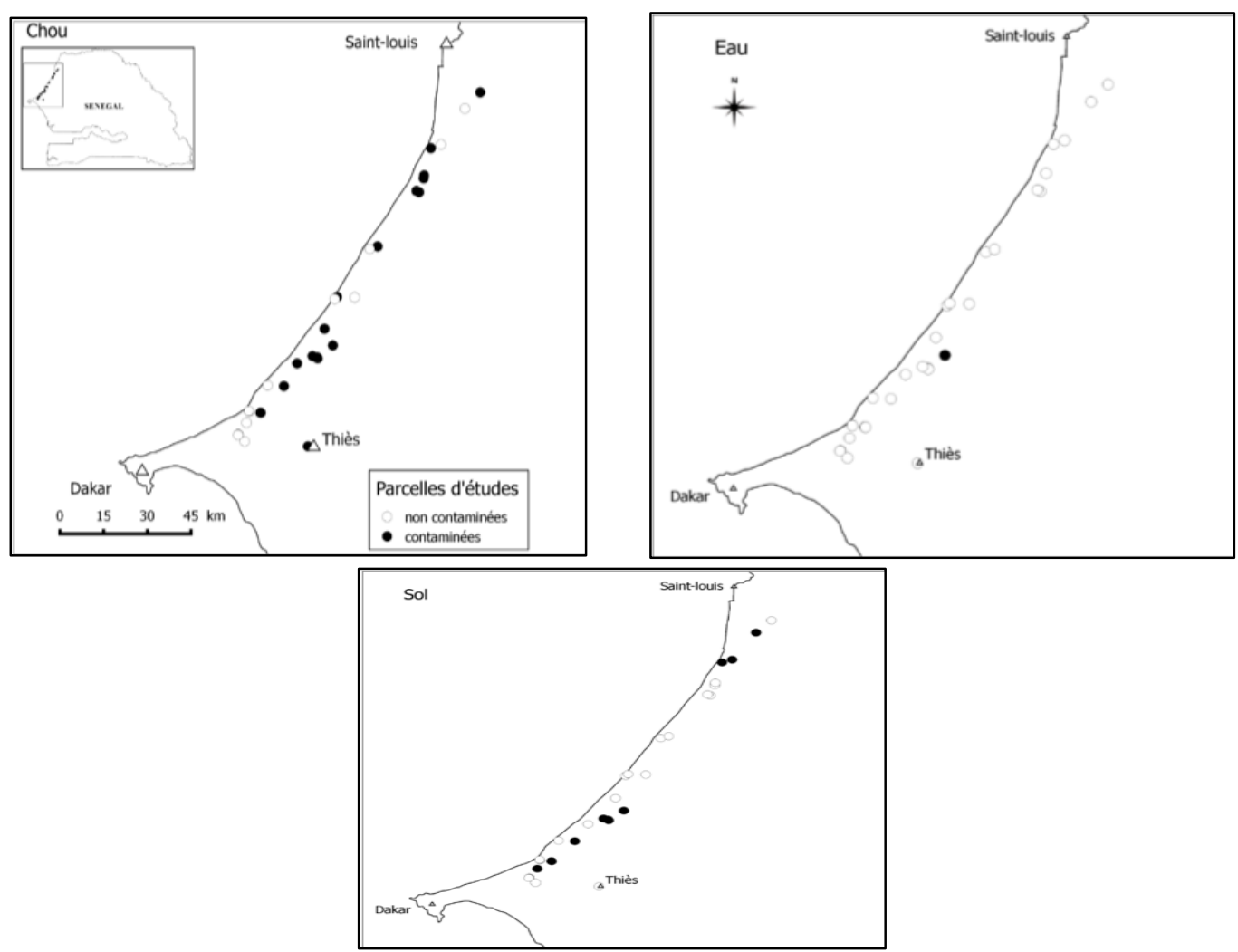

Figure 2 : Contamination des matrices environnementales par les pesticides dans la zone des Niayes. Chou : matrice chou (30 parcelles suivant 5 pieds/parcelle); Sol : matrice sol (30 parcelles suivant $1 \mathrm{~kg} / \mathrm{parcelle})$; Eau : matrice eau (30 parcelles suivant 2,5 litres/parcelle) 
Tableau 1 : Teneur en résidus de pesticide dans les échantillons d'eau prélevés au niveau des puits et des « céanes » des parcelles maraichères dans la zone des Niayes. 30 puits ou « céanes » suivant 2,5 litres/puits. Les teneurs des résidus sont exprimées en $\mu \mathrm{g} / \mathrm{L}$.

\begin{tabular}{|c|c|c|c|c|c|c|c|c|c|c|c|c|c|c|c|c|c|c|c|}
\hline & $\frac{O}{3}$ & 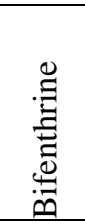 & 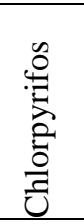 & 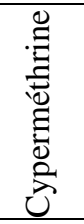 & 合 & 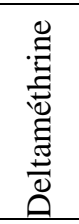 & $\begin{array}{l}\overline{0} \\
0 \\
.0 \\
0\end{array}$ & $\begin{array}{l}\stackrel{\Xi}{\Xi} \\
\stackrel{\Xi}{\Xi} \\
\ddot{\theta}\end{array}$ & 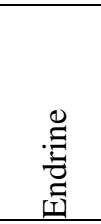 & 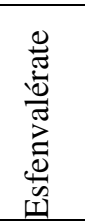 & 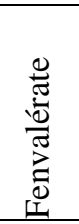 & $\begin{array}{l}\frac{0}{0} \\
\frac{0}{0} \\
\frac{\pi}{0} \\
\frac{2}{2}\end{array}$ & 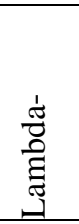 & 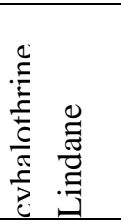 & 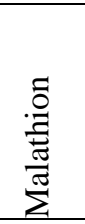 & 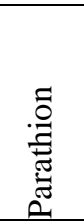 & $\begin{array}{l}\frac{0}{0} \\
0 \\
\frac{1}{0} \\
0 \\
0\end{array}$ & : & 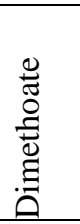 \\
\hline Bayakh & $<0,03^{1}$ & $<0,1$ & $<0,1$ & $<0,1$ & $<0,1$ & $<0,1$ & $<0,1$ & $<0,03$ & $<0,03$ & $<0,1$ & $<0,1$ & $<0,03$ & $<0,1$ & $<0,1$ & $<0,1$ & $<0,1$ & $<0,1$ & $<0,1$ & $<0,1$ \\
\hline Dara & $2<0,03$ & $<0,1$ & $<0,1$ & $<0,1$ & $<0,1$ & $<0,1$ & $<0,1$ & $<0,03$ & $<0,03$ & $<0,1$ & $<0,1$ & $<0,03$ & $<0,1$ & $<0,1$ & $<0,1$ & $<0,1$ & $<0,1$ & $<0,1$ & $<0,1$ \\
\hline Darou Khoudoss/Diogo & $<0,03$ & $<0,1$ & $<0,1$ & $<0,1$ & $<0,1$ & $<0,1$ & $<0,1$ & $<0,03$ & $<0,03$ & $<0,1$ & $<0,1$ & $<0,03$ & $<0,1$ & $<0,1$ & $<0,1$ & $<0,1$ & $<0,1$ & $<0,1$ & $<0,1$ \\
\hline Darou Khoudoss/Diogo & $<0,03$ & $<0,1$ & $<0,1$ & $<0,1$ & $<0,1$ & $<0,1$ & $<0,1$ & $<0,03$ & $<0,03$ & $<0,1$ & $<0,1$ & $<0,03$ & $<0,1$ & $<0,1$ & $<0,1$ & $<0,1$ & $<0,1$ & $<0,1$ & $<0,1$ \\
\hline Fassboye/Lite & $<0,03$ & $<0,1$ & $<0,1$ & $<0,1$ & $<0,1$ & $<0,1$ & $<0,1$ & $<0,03$ & $<0,03$ & $<0,1$ & $<0,1$ & $<0,03$ & $<0,1$ & $<0,1$ & $<0,1$ & $<0,1$ & $<0,1$ & $<0,1$ & $<0,1$ \\
\hline Fassboye/Lite & $<0,03$ & $<0,1$ & $<0,1$ & $<0,1$ & $<0,1$ & $<0,1$ & $<0,1$ & $<0,03$ & $<0,03$ & $<0,1$ & $<0,1$ & $<0,03$ & $<0,1$ & $<0,1$ & $<0,1$ & $<0,1$ & $<0,1$ & $<0,1$ & $<0,1$ \\
\hline Gorom 1 & $<0,03$ & $<0,1$ & $<0,1$ & $<0,1$ & $<0,1$ & $<0,1$ & $<0,1$ & $<0,03$ & $<0,03$ & $<0,1$ & $<0,1$ & $<0,03$ & $<0,1$ & $<0,1$ & $<0,1$ & $<0,1$ & $<0,1$ & $<0,1$ & $<0,1$ \\
\hline Gorom 1 & $<0,03$ & $<0,1$ & $<0,1$ & $<0,1$ & $<0,1$ & $<0,1$ & $<0,1$ & $<0,03$ & $<0,03$ & $<0,1$ & $<0,1$ & $<0,03$ & $<0,1$ & $<0,1$ & $<0,1$ & $<0,1$ & $<0,1$ & $<0,1$ & $<0,1$ \\
\hline Kalassane- Ndoyediagne & $<0,03$ & $<0,1$ & $<0,1$ & $<0,1$ & $<0,1$ & $<0,1$ & $<0,1$ & $<0,03$ & $<0,03$ & $<0,1$ & $<0,1$ & $<0,03$ & $<0,1$ & $<0,1$ & $<0,1$ & $<0,1$ & $<0,1$ & $<0,1$ & $<0,1$ \\
\hline Kalassane- Ndoyediagne & $<0,03$ & $<0,1$ & $<0,1$ & $<0,1$ & $<0,1$ & $<0,1$ & $<0,1$ & $<0,03$ & $<0,03$ & $<0,1$ & $<0,1$ & $<0,03$ & $<0,1$ & $<0,1$ & $<0,1$ & $<0,1$ & $<0,1$ & $<0,1$ & $<0,1$ \\
\hline Kayar-Kan & $<0,03$ & $<0,1$ & $<0,1$ & $<0,1$ & $<0,1$ & $<0,1$ & $<0,1$ & $<0,03$ & $<0,03$ & $<0,1$ & $<0,1$ & $<0,03$ & $<0,1$ & $<0,1$ & $<0,1$ & $<0,1$ & $<0,1$ & $<0,1$ & $<0,1$ \\
\hline Kayar-Kan & $<0,03$ & $<0,1$ & $<0,1$ & $<0,1$ & $<0,1$ & $<0,1$ & $<0,1$ & $<0,03$ & $<0,03$ & $<0,1$ & $<0,1$ & $<0,03$ & $<0,1$ & $<0,1$ & $<0,1$ & $<0,1$ & $<0,1$ & $<0,1$ & $<0,1$ \\
\hline \multicolumn{20}{|l|}{ Konqueyoye- Lompoul/ } \\
\hline Mer & $<0,03$ & $<0,1$ & $<0,1$ & $<0,1$ & $<0,1$ & $<0,1$ & $<0,1$ & $<0,03$ & $<0,03$ & $<0,1$ & $<0,1$ & $<0,03$ & $<0,1$ & $<0,1$ & $<0,1$ & $<0,1$ & $<0,1$ & $<0,1$ & $<0,1$ \\
\hline
\end{tabular}




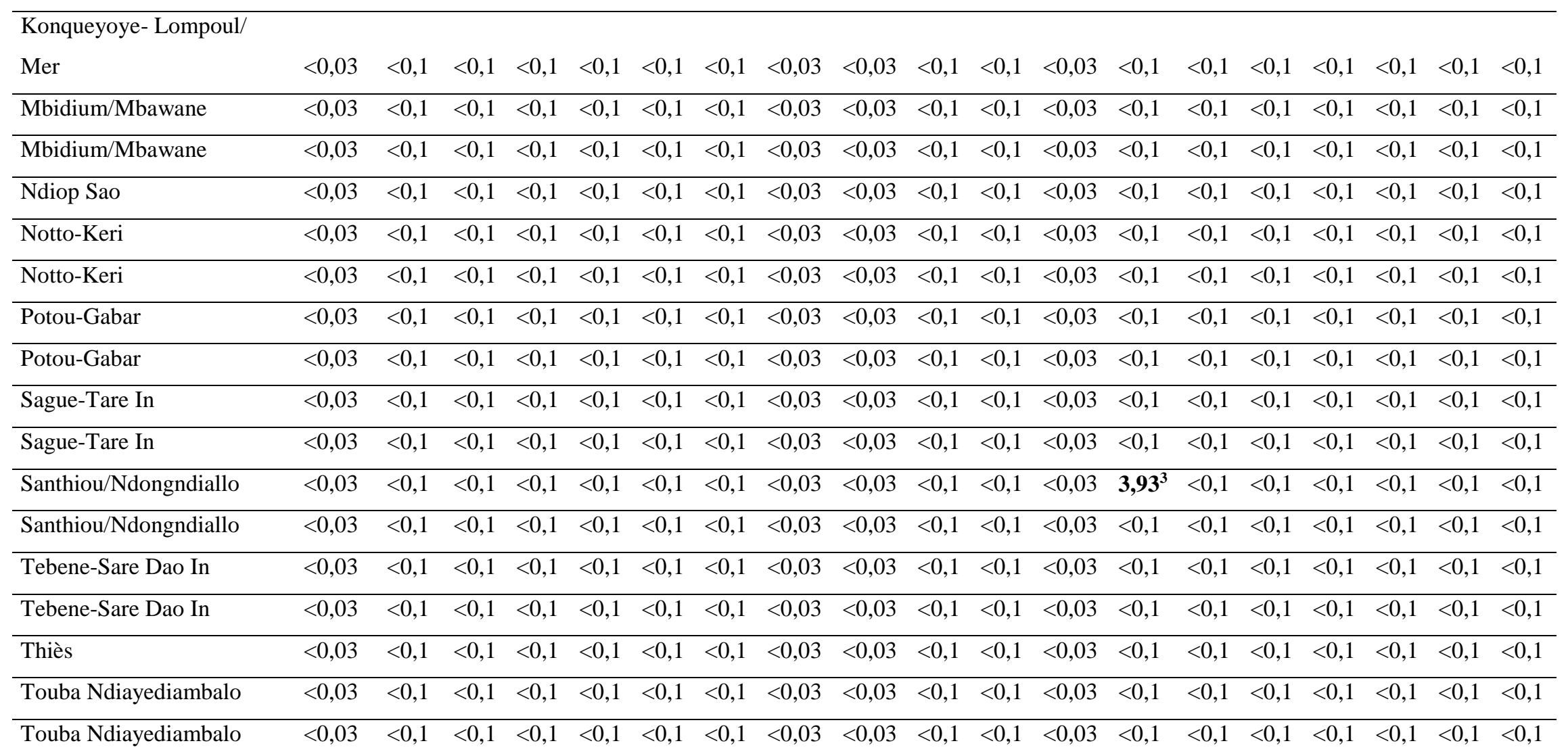

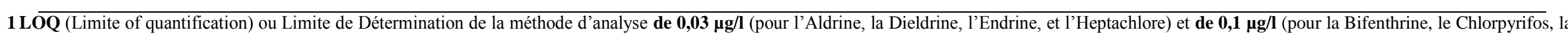
Cyperméthrine, le DDT, la Deltaméthrine, le Dicofol, l'Esfenvalérate, le Fenvalérate, la Lambda-Cyhalothrine, le Lindane, le Malathion, le Parathion, le Profénofos, la Trifluraline et le Diméthoate)

$2<\mathrm{LOQ}$ signifiant que la matière active de pesticide recherchée n'est pas présente

3Valeur en gras lorsqu'elle est supérieure à norme de l'OMS de l'eau potable selon les Directives de qualité pour l'eau de boisson, quatrième édition, intégrant le premier additif (OMS 2017) 

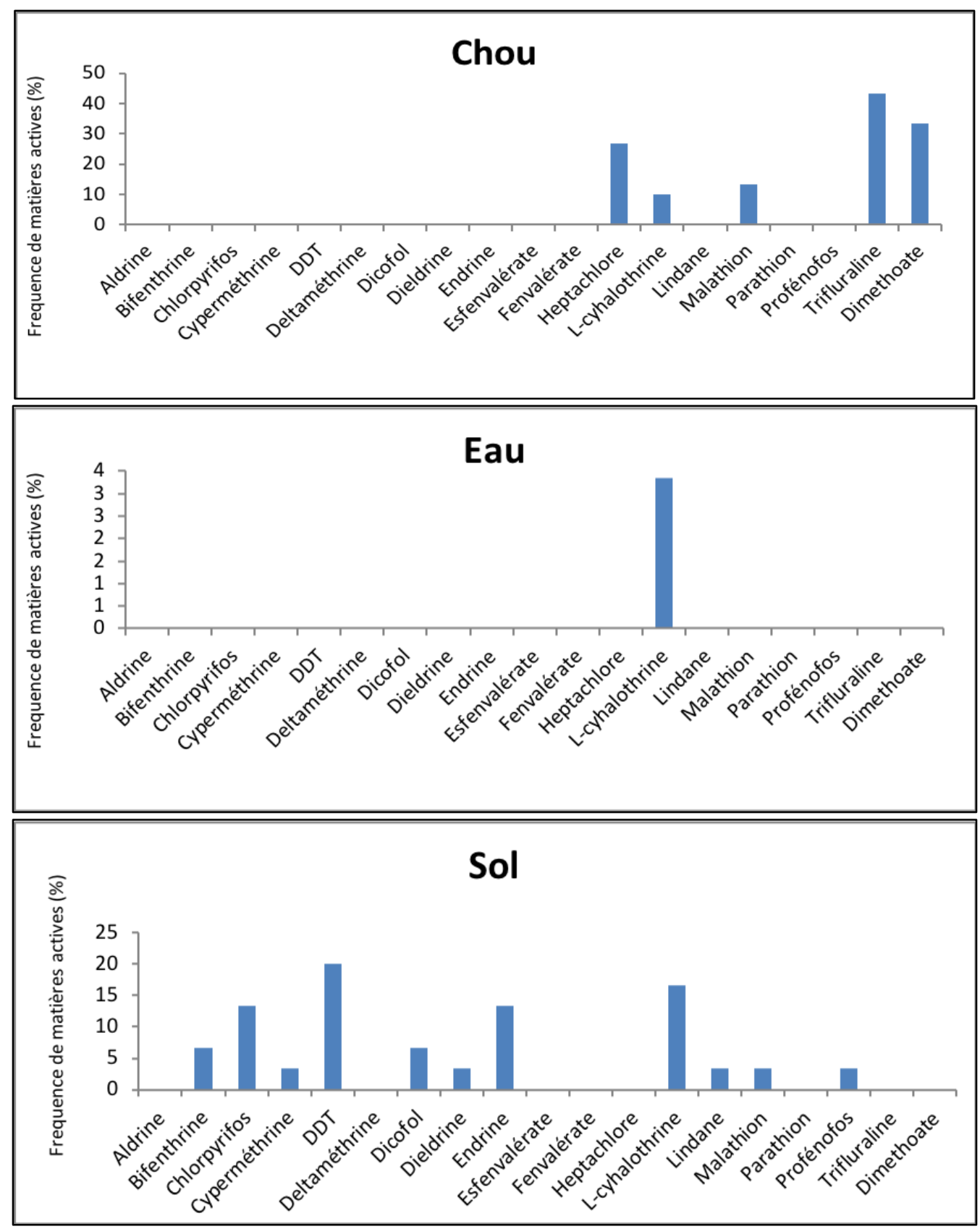

Figure 3 : Fréquence des matières actives dans les matrices environnementales dans la zone des Niayes. Chou : matrice chou (30 parcelles suivant 5 pieds/parcelle) ; Eau : matrice eau (30 puits ou « céanes » suivant 2,5 litres/puits) ; Sol : matrice sol (30 parcelles suivant $1 \mathrm{~kg} /$ parcelle). 
Tableau 2 : Teneur en résidus de pesticide dans les échantillons de sol prélevés au niveau des parcelles maraichères dans la zone des Niayes (en $\mu \mathrm{g} / \mathrm{kg}$ ). 30 parcelles suivant $1 \mathrm{~kg} /$ parcelle. Les teneurs des résidus sont exprimées en $\mu \mathrm{g} / \mathrm{kg}$.

\begin{tabular}{|c|c|c|c|c|c|c|c|c|c|c|c|c|c|c|c|c|c|c|c|}
\hline & 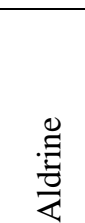 & 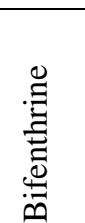 & 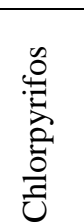 & 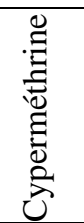 & 穴 & 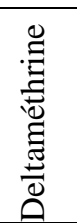 & $\begin{array}{l}\overrightarrow{0} \\
\stackrel{0}{0} \\
\stackrel{0}{0}\end{array}$ & 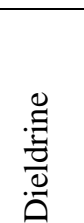 & 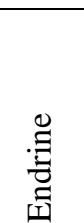 & 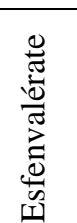 & 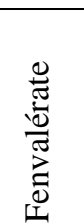 & $\begin{array}{l}\frac{0}{0} \\
\frac{0}{0} \\
\frac{\pi}{2} \\
\frac{0}{2}\end{array}$ & 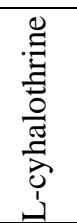 & 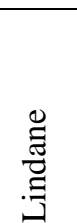 & 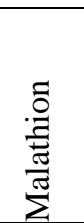 & 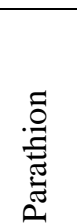 & 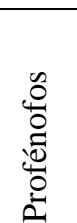 & 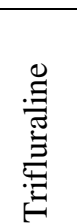 & 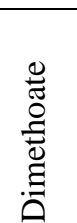 \\
\hline Bayakh & $<10^{1}$ & $\mathbf{7 3}^{3}$ & $<10$ & $<10$ & $\overline{19}$ & $<10$ & $<10$ & $<10$ & 613 & $<10$ & $<10$ & $<10$ & $<10$ & $<10$ & $<10$ & $<10$ & $<10$ & $<10$ & $<0,1$ \\
\hline Dara & $2<10$ & $<10$ & $<10$ & $<10$ & $<10$ & $<10$ & $<10$ & $<10$ & $<10$ & $<10$ & $<10$ & $<10$ & $<10$ & $<10$ & $<10$ & $<10$ & $<10$ & $<10$ & $<0,1$ \\
\hline Darou khoudoss/diogo & $<10$ & $<10$ & $<10$ & 71 & $<10$ & $<10$ & 40 & 30 & $<10$ & $<10$ & $<10$ & $<10$ & $<10$ & $<10$ & $<10$ & $<10$ & $<10$ & $<10$ & $<0,1$ \\
\hline Darou khoudoss/diogo & $<10$ & $<10$ & $<10$ & $<10$ & 74 & $<10$ & $<10$ & $<10$ & $<10$ & $<10$ & $<10$ & $<10$ & $<10$ & $<10$ & $<10$ & $<10$ & $<10$ & $<10$ & $<0,1$ \\
\hline Fassboye/Lite & $<10$ & $<10$ & $<10$ & $<10$ & $<10$ & $<10$ & $<10$ & $<10$ & $<10$ & $<10$ & $<10$ & $<10$ & $<10$ & $<10$ & $<10$ & $<10$ & $<10$ & $<10$ & $<0,1$ \\
\hline Fassboye/Lite & $<10$ & $<10$ & $<10$ & $<10$ & $<10$ & $<10$ & $<10$ & $<10$ & $<10$ & $<10$ & $<10$ & $<10$ & $<10$ & $<10$ & $<10$ & $<10$ & $<10$ & $<10$ & $<0,1$ \\
\hline Gorom 1 & $<10$ & $<10$ & $<10$ & $<10$ & $<10$ & $<10$ & 13 & $<10$ & $<10$ & $<10$ & $<10$ & $<10$ & 49 & $<10$ & $<10$ & $<10$ & $<10$ & $<10$ & $<0,1$ \\
\hline Gorom 1 & $<10$ & $<10$ & $<10$ & $<10$ & $<10$ & $<10$ & $<10$ & $<10$ & $<10$ & $<10$ & $<10$ & $<10$ & $<10$ & $<10$ & $<10$ & $<10$ & $<10$ & $<10$ & $<0,1$ \\
\hline Kalassane- ndoyediagne & $<10$ & $<10$ & $<10$ & $<10$ & $<10$ & $<10$ & $<10$ & $<10$ & $<10$ & $<10$ & $<10$ & $<10$ & 25 & $<10$ & $<10$ & $<10$ & $<10$ & $<10$ & $<0,1$ \\
\hline Kalassane- ndoyediagne & $<10$ & $<10$ & $<10$ & $<10$ & $<10$ & $<10$ & $<10$ & $<10$ & $<10$ & $<10$ & $<10$ & $<10$ & 18 & $<10$ & $<10$ & $<10$ & $<10$ & $<10$ & $<0,1$ \\
\hline Kayar-kan & $<10$ & $<10$ & 19 & $<10$ & 163 & $<10$ & $<10$ & $<10$ & 88 & $<10$ & $<10$ & $<10$ & $<10$ & $<10$ & $<10$ & $<10$ & $<10$ & $<10$ & $<0,1$ \\
\hline Kayar-kan & $<10$ & $<10$ & $<10$ & $<10$ & $<10$ & $<10$ & $<10$ & $<10$ & $<10$ & $<10$ & $<10$ & $<10$ & $<10$ & $<10$ & $<10$ & $<10$ & $<10$ & $<10$ & $<0,1$ \\
\hline Konqueyoye- lompoul/ mer & $<10$ & $<10$ & $<10$ & $<10$ & $<10$ & $<10$ & $<10$ & $<10$ & $<10$ & $<10$ & $<10$ & $<10$ & $<10$ & $<10$ & $<10$ & $<10$ & $<10$ & $<10$ & $<0,1$ \\
\hline Konqueyoye- lompoul/ mer & $<10$ & $<10$ & $<10$ & $<10$ & $<10$ & $<10$ & $<10$ & $<10$ & $<10$ & $<10$ & $<10$ & $<10$ & $<10$ & $<10$ & $<10$ & $<10$ & $<10$ & $<10$ & $<0,1$ \\
\hline Mbidium/Mbawane & $<10$ & $<10$ & $<10$ & $<10$ & 15 & $<10$ & $<10$ & $<10$ & $<10$ & $<10$ & $<10$ & $<10$ & 316 & $<10$ & $<10$ & $<10$ & $<10$ & $<10$ & $<0,1$ \\
\hline
\end{tabular}


P. S. GUEYE et al. / Int. J. Biol. Chem. Sci. 14(2): 539-554, 2020

\begin{tabular}{|c|c|c|c|c|c|c|c|c|c|c|c|c|c|c|c|c|c|c|c|}
\hline Mbidium/Mbawane & $<10$ & $<10$ & $<10$ & $<10$ & 114 & $<10$ & $<10$ & $<10$ & $<10$ & $<10$ & $<10$ & $<10$ & $<10$ & $<10$ & $<10$ & $<10$ & $<10$ & $<10$ & $<0,1$ \\
\hline Ndiop Sao & $<10$ & $<10$ & $<10$ & $<10$ & $<10$ & $<10$ & $<10$ & $<10$ & $<10$ & $<10$ & $<10$ & $<10$ & $<10$ & $<10$ & $<10$ & $<10$ & $<10$ & $<10$ & $<0,1$ \\
\hline Notto-Keri & $<10$ & $<10$ & 19 & $<10$ & 17 & $<10$ & $<10$ & $<10$ & 12 & $<10$ & $<10$ & $<10$ & $<10$ & 15 & $<10$ & $<10$ & $<10$ & $<10$ & $<0,1$ \\
\hline Notto-Keri & $<10$ & $<10$ & $<10$ & $<10$ & $<10$ & $<10$ & $<10$ & $<10$ & $<10$ & $<10$ & $<10$ & $<10$ & $<10$ & $<10$ & $<10$ & $<10$ & $<10$ & $<10$ & $<0,1$ \\
\hline Potou-gabar & $<10$ & $<10$ & 17 & $<10$ & $<10$ & $<10$ & $<10$ & $<10$ & $<10$ & $<10$ & $<10$ & $<10$ & $<10$ & $<10$ & $<10$ & $<10$ & $<10$ & $<10$ & $<0,1$ \\
\hline Potou-gabar & $<10$ & $<10$ & $<10$ & $<10$ & $<10$ & $<10$ & $<10$ & $<10$ & $<10$ & $<10$ & $<10$ & $<10$ & $<10$ & $<10$ & $<10$ & $<10$ & $<10$ & $<10$ & $<0,1$ \\
\hline Sague-tare in & $<10$ & $<10$ & $<10$ & $<10$ & $<10$ & $<10$ & $<10$ & $<10$ & $<10$ & $<10$ & $<10$ & $<10$ & $<10$ & $<10$ & $<10$ & $<10$ & $<10$ & $<10$ & $<0,1$ \\
\hline Sague-tare in & $<10$ & $<10$ & $<10$ & $<10$ & $<10$ & $<10$ & $<10$ & $<10$ & $<10$ & $<10$ & $<10$ & $<10$ & $<10$ & $<10$ & $<10$ & $<10$ & $<10$ & $<10$ & $<0,1$ \\
\hline Santhiou/ndongndiallo & $<10$ & $<10$ & 21 & $<10$ & $<10$ & $<10$ & $<10$ & $<10$ & 63 & $<10$ & $<10$ & $<10$ & 46 & $<10$ & 209 & $<10$ & 235 & $<10$ & $<0,1$ \\
\hline Santhiou/ndongndiallo & $<10$ & $<10$ & $<10$ & $<10$ & $<10$ & $<10$ & $<10$ & $<10$ & $<10$ & $<10$ & $<10$ & $<10$ & $<10$ & $<10$ & $<10$ & $<10$ & $<10$ & $<10$ & $<0,1$ \\
\hline Tebene-sare dao in & $<10$ & $<10$ & $<10$ & $<10$ & $<10$ & $<10$ & $<10$ & $<10$ & $<10$ & $<10$ & $<10$ & $<10$ & $<10$ & $<10$ & $<10$ & $<10$ & $<10$ & $<10$ & $<0,1$ \\
\hline Tebene-sare dao in & $<10$ & $<10$ & $<10$ & $<10$ & $<10$ & $<10$ & $<10$ & $<10$ & $<10$ & $<10$ & $<10$ & $<10$ & $<10$ & $<10$ & $<10$ & $<10$ & $<10$ & $<10$ & $<0,1$ \\
\hline Thiès & $<10$ & $<10$ & $<10$ & $<10$ & $<10$ & $<10$ & $<10$ & $<10$ & $<10$ & $<10$ & $<10$ & $<10$ & $<10$ & $<10$ & $<10$ & $<10$ & $<10$ & $<10$ & $<0,1$ \\
\hline Touba ndiayediambalo & $<10$ & 16 & $<10$ & $<10$ & $<10$ & $<10$ & $<10$ & $<10$ & $<10$ & $<10$ & $<10$ & $<10$ & $<10$ & $<10$ & $<10$ & $<10$ & $<10$ & $<10$ & $<0,1$ \\
\hline Touba ndiayediambalo & $<10$ & $<10$ & $<10$ & $<10$ & $<10$ & $<10$ & $<10$ & $<10$ & $<10$ & $<10$ & $<10$ & $<10$ & $<10$ & $<10$ & $<10$ & $<10$ & $<10$ & $<10$ & $<0,1$ \\
\hline
\end{tabular}

$\mathbf{1} \mathbf{L O Q}$ (Limite of quantification) ou Limite de Détermination de la méthode d'analyse de $\mathbf{1 0} \boldsymbol{\mu g} / \mathbf{k g}$ (pour Aldrine, Bifenthrine Chlorpyrifos, Cyperméthrine, DDT, Deltaméthrine, Dicofol, Dieldrine, Endrine, Esfenvalérate, Fenvalérate, Heptachlore, Lambda-cyhalothrine, Lindane, Malathion, Parathion, Profénofos et Trifluraline) et de 0,1 $\boldsymbol{\mu g} / \mathbf{k g}$ pour le Diméthoate.

$\mathbf{2}<$ LOQ de la méthode d'analyse signifiant que la matière active de pesticide recherchée n'est pas présente

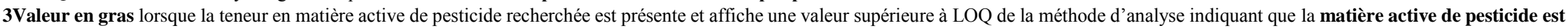
bien présente 
Tableau 3 : Teneur en résidus de pesticide dans les échantillons de chou prélevés au niveau des parcelles maraichères dans la zone des Niayes. 30 parcelles suivant 5 pieds/parcelle. Les teneurs des résidus sont exprimées en $\mathrm{mg} / \mathrm{kg}$.

\begin{tabular}{|c|c|c|c|c|c|c|c|c|c|c|c|c|c|c|c|c|c|c|c|}
\hline & 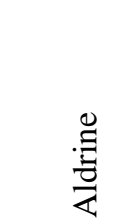 & 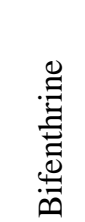 & 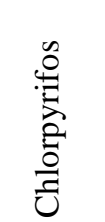 & 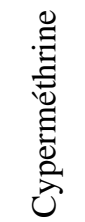 & 容 & 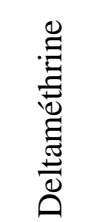 & $\begin{array}{l}\overline{0} \\
0 \\
.0 \\
0\end{array}$ & 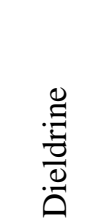 & 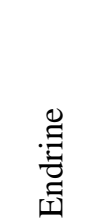 & 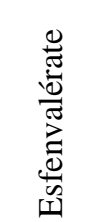 & 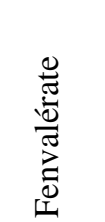 & $\begin{array}{l}\frac{0}{0} \\
\frac{0}{0} \\
\frac{\pi}{2} \\
\frac{0}{2}\end{array}$ & 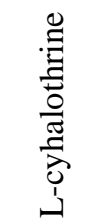 & 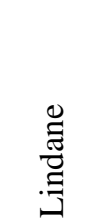 & 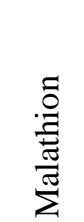 & 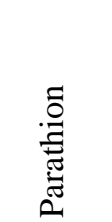 & 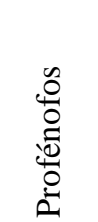 & 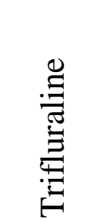 & 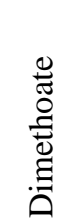 \\
\hline Bayakh & $<0,01^{1}$ & $<0,1$ & $<0,05$ & $<0,1$ & $<0,01$ & $<0,05$ & $<0,01$ & $<0,01$ & $<0,01$ & $<0,05$ & $<0,01$ & $0,21^{3}$ & $<0,05$ & $<0,01$ & $<0,1$ & $<0,01$ & $<0,01$ & $<0,01$ & 4,02 \\
\hline Dara & $2<0,01$ & $<0,1$ & $<0,05$ & $<0,1$ & $<0,01$ & $<0,05$ & $<0,01$ & $<0,01$ & $<0,01$ & $<0,05$ & $<0,01$ & $<0,01$ & $<0,05$ & $<0,01$ & $<0,1$ & $<0,01$ & $<0,01$ & $<0,01$ & $<0,1$ \\
\hline Darou khoudoss/diogo & $<0,01$ & $<0,1$ & $<0,05$ & $<0,1$ & $<0,01$ & $<0,05$ & $<0,01$ & $<0,01$ & $<0,01$ & $<0,05$ & $<0,01$ & $\mathbf{0 , 0 3}$ & $<0,05$ & $<0,01$ & $<0,1$ & $<0,01$ & $<0,01$ & 0,07 & 1,47 \\
\hline Fassboye/Lite & $<0,01$ & $<0,1$ & $<0,05$ & $<0,1$ & $<0,01$ & $<0,05$ & $<0,01$ & $<0,01$ & $<0,01$ & $<0,05$ & $<0,01$ & $<0,01$ & $<0,05$ & $<0,01$ & $\mathbf{0 , 3 0}$ & $<0,01$ & $<0,01$ & $<0,01$ & 1,26 \\
\hline Fassboye/Lite & $<0,01$ & $<0,1$ & $<0,05$ & $<0,1$ & $<0,01$ & $<0,05$ & $<0,01$ & $<0,01$ & $<0,01$ & $<0,05$ & $<0,01$ & $<0,01$ & $<0,05$ & $<0,01$ & $<0,1$ & $<0,01$ & $<0,01$ & $\mathbf{0 , 0 9}$ & $<0,1$ \\
\hline Gorom 1 & $<0,01$ & $<0,1$ & $<0,05$ & $<0,1$ & $<0,01$ & $<0,05$ & $<0,01$ & $<0,01$ & $<0,01$ & $<0,05$ & $<0,01$ & $<0,01$ & 0,11 & $<0,01$ & $<0,1$ & $<0,01$ & $<0,01$ & $<0,01$ & $<0,1$ \\
\hline Gorom 1 & $<0,01$ & $<0,1$ & $<0,05$ & $<0,1$ & $<0,01$ & $<0,05$ & $<0,01$ & $<0,01$ & $<0,01$ & $<0,05$ & $<0,01$ & $<0,01$ & $<0,05$ & $<0,01$ & $<0,1$ & $<0,01$ & $<0,01$ & $<0,01$ & $<0,1$ \\
\hline Kayar-kan & $<0,01$ & $<0,1$ & $<0,05$ & $<0,1$ & $<0,01$ & $<0,05$ & $<0,01$ & $<0,01$ & $<0,01$ & $<0,05$ & $<0,01$ & $<0,01$ & $<0,05$ & $<0,01$ & $<0,1$ & $<0,01$ & $<0,01$ & 0,09 & $<0,1$ \\
\hline Konqueyoye- lompoul/ mer & $<0,01$ & $<0,1$ & $<0,05$ & $<0,1$ & $<0,01$ & $<0,05$ & $<0,01$ & $<0,01$ & $<0,01$ & $<0,05$ & $<0,01$ & $<0,01$ & $<0,05$ & $<0,01$ & $<0,1$ & $<0,01$ & $<0,01$ & $<0,01$ & 1,71 \\
\hline Konqueyoye- lompoul/ mer & $<0,01$ & $<0,1$ & $<0,05$ & $<0,1$ & $<0,01$ & $<0,05$ & $<0,01$ & $<0,01$ & $<0,01$ & $<0,05$ & $<0,01$ & $<0,01$ & $<0,05$ & $<0,01$ & $<0,1$ & $<0,01$ & $<0,01$ & $<0,01$ & $<0,1$ \\
\hline Mbidium/Mbawane & $<0,01$ & $<0,1$ & $<0,05$ & $<0,1$ & $<0,01$ & $<0,05$ & $<0,01$ & $<0,01$ & $<0,01$ & $<0,05$ & $<0,01$ & $\mathbf{0 , 3 3}$ & $<0,05$ & $<0,01$ & $<0,1$ & $<0,01$ & $<0,01$ & $<0,01$ & $<0,1$ \\
\hline
\end{tabular}


P. S. GUEYE et al. / Int. J. Biol. Chem. Sci. 14(2): 539-554, 2020

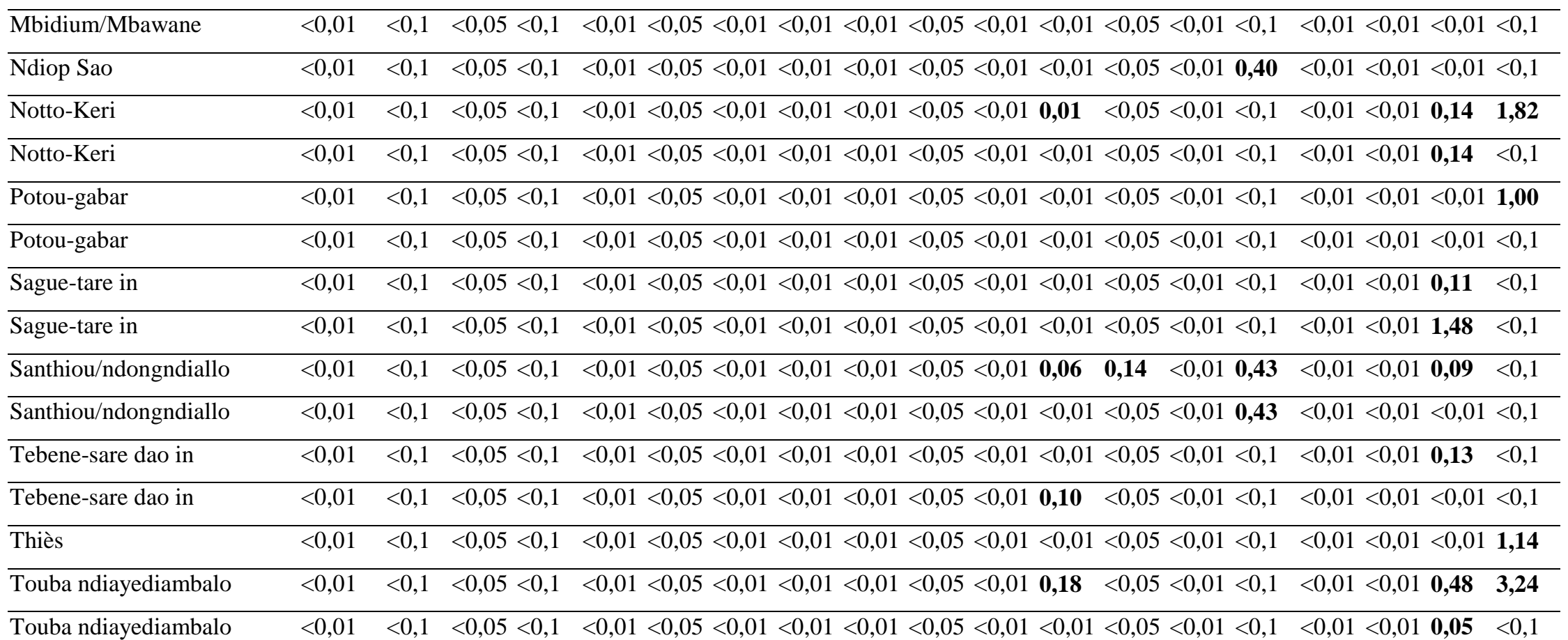

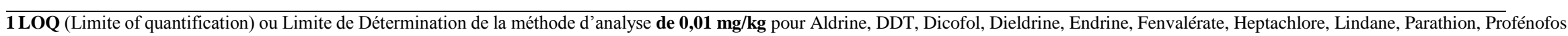
et Trifluraline, de $\mathbf{0 , 0 5} \mathbf{~ m g} / \mathbf{k g}$ pour Chlorpyrifos, Deltaméthrine, Esfenvalérate et Lambda-cyhalothrine et de 0,1 mg/kg pour Cyperméthrine, Malathion, Bifenthrine et Diméthoate.

$2<$ LOQ de la méthode d'analyse signifiant que la matière active de pesticide recherchée n'est pas présente

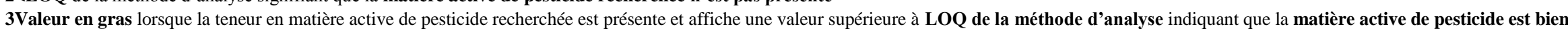
présente 


\section{DISCUSSION}

\section{Contamination des eaux de puits et de "céanes" par les pesticides}

$\mathrm{La}$ lambda-cyhalothrine est une pyréthrinoïde modérément toxique qui se dégrade rapidement à la lumière. Au Sénégal, plusieurs études ont montré que la contamination par les pesticides des eaux d'arrosage (puits et "céanes") est fréquente (Ngom et al., 2012; Diop et al., 2016). La même situation a été observée au niveau de la sous-région avec des produits plus dangereux. En effet, une étude menée dans la ville de Lomé au Togo, montre que les eaux d'arrosage et de boisson (robinet et puits) présentent des niveaux de résidus d'aldrine, de dieldrine et d'heptachlore 43 fois supérieurs aux normes de l'Union Européenne et de l'OMS (Mawussi, 2008). Les pesticides organochlorés (endosulfan et hexachlorocyclohexane) étaient détectés avec des teneurs cumulées pouvant atteindre 25,63 $\mu \mathrm{g} / \mathrm{L}$ par endroit en Côte d'Ivoire (Traoré et al., 2006). Le résultat sur la situation de référence de la contamination par les pesticides de l'eau au Sénégal s'expliquerait par la maitrise des bonnes pratiques phytosanitaires (Ngom et al., 2012; Ngom et al., 2013). Les principaux facteurs de contamination par les pesticides de l'eau seraient liés au lessivage des sols de culture par les eaux de ruissellement, la perméabilité des sols avec l'infiltration rapide des eaux de pluies et d'arrosage, la fréquence élevée des traitements, le rejet incontrôlé des emballages vides et la mauvaise gestion des effluents phytosanitaires.

\section{Contamination des sols par les pesticides}

Les pesticides trouvés appartiennent à deux familles de pesticides qui sont les organochlorés et les pyréthrinoïdes. Les organochlorés trouvés sont des Polluants organiques persistants (POP) contrairement aux pyréthrinoïdes (Régnault, 2005). La contamination des zones centre et sud serait liée à la forte activité maraichère pour le contrôle des ravageurs et à la proximité des marchés d'approvisionnement en pesticides (Diop,
2013). Par ailleurs, des sols contaminés par les mêmes familles de pesticides ont été observés en Côte d'Ivoire (MAMBE-ANI et al., 2019), au Togo (Mawussi, 2008), au Benin (AssogbaKomlan et al., 2007), et au Mali (Dem et al.,2007). La présence des POP (DDT et lindane) s'expliquerait par l'accès frauduleux à ces produits et leur caractère persistant (Ngom et al., 2012; Ngom et al., 2013). La contamination par les pyréthrinoïdes (lambdacyhalothrine) et les organophosphorés (chlorpyrifos) serait liée à la fréquence de leur utilisation par les maraichers. La faible présence $\mathrm{du}$ lindane (POP), du profénopfos et du malathion (Organophosphoré) et de la cyperméthrine (pyréthrinoïde) pourrait s'expliquer par leur faible disponibilité.

Les sols par leurs niveaux de contaminations élevés constitueraient une source de dissémination de la contamination vers les autres compartiments de l'environnement et les produits agricoles.

\section{Contamination du chou par les pesticides}

La forte contamination du chou dans la zone centre pourrait s'expliquer par la disponibilité et la fréquence d'application des pesticides. Au regard des résultats obtenus, l'eau et le sol ne sont pas des sources de contamination du chou. Les matières actives présentes dans l'eau et sol n'ont pas été observées sur le chou. La présence du malathion à la fois sur le sol et sur le chou est due à une contamination directe liée aux pratiques maraichères (Cissé et al., 2008 ; Samake et al., 2011 ; Ngom et al., 2013 ; Diop et al., 2016 ; Soro et al., 2019). L'heptachlore (POP), produit non homologué au Sénégal, contamine le chou (Soumaré CH, 2008). Cela prouve que la contamination des produits maraichers est liée à l'utilisation des pesticides par les producteurs. En effet, les produits qui contaminent le chou n'ont pas été observés dans les matrices environnementales eau et sol.

\section{Conclusion}

Cette étude dont l'objectif est d'identifier les sources de contamination du 
chou à travers l'évaluation du niveau de contamination des matrices nous a permis de montrer que les matrices environnementales, eau et sol, sont contaminées mais elles ne sont pas les sources de contamination du chou. L'heptachlore (POP), produit non homologué au Sénégal, contamine le chou. Cela prouve que la contamination des produits maraichers est liée à l'utilisation des pesticides par les producteurs. En effet, les produits qui contaminent le chou n'ont pas été observés dans les matrices environnementales eau et sol. Ce travail a fourni des informations intéressantes sur les risques d'exposition liés aux résidus de pesticides au Sénégal à travers les pratiques agricoles. Mais également la disponibilité de produits non homologués à travers un approvisionnement frauduleux. Il ébauche un référentiel pour la mise au point d'un outil d'aide à la décision indispensable pour une meilleure gestion des pesticides qu'il faudra affiner et partager dans la perspective d'une bonne gestion de la qualité sanitaire du chou relativement aux résidus de pesticides.

\section{CONFLIT D'INTÉRÊTS}

Les auteurs déclarent qu'il n'existe aucun conflit d'intérêts.

\section{CONTRIBUTIONS DES AUTEURS}

PSG a été l'investigateur principal; BL, MD et KD ont co-encadré les travaux de terrain et ont participé à la rédaction ; BL a participé à la mise en place du protocole et à la rédaction du manuscrit.

\section{REMERCIEMENTS}

Nous tenons à remercier le Centre Régional de Recherches en Ecotoxicologie et Sécurité Environnementale (CERES-Locustox) pour avoir soutenu financièrement la réalisation de cette étude, le Laboratoire Production et Protection Intégrées en Agroécosystème (L2PIA) de l'Université Cheikh Anta Diop de Dakar (UCAD) pour l'encadrement et la supervision de ce travail et la Fédération des Producteurs Maraîchers de la zone des Niayes
(FMPN) pour son aide dans la facilitation des échantillonnages.

\section{REFERENCES}

ANDS (Agence Nationale de la Démographie et de la Statistique). 2013. Situation Économique et Sociale du Sénégal en 2011. 109pp.

Assogba-Komlan F, Anihouvi P, Achigan E, Sikirou R, Boko A, Adje C, Ahle V, Vodouhe R, Assa A. 2007. Pratiques culturales et teneur en éléments anti nutritionnels (nitrates et pesticides) du Solanum macrocarpum au sud du Bénin. Afr. J. Food Agric. Nutr. Dev., 7: 1-21. https://www.ajol.info/index.php/ajfand/ar ticle/download/136155/125646

Cissé I, Badiane M, Ngom S, Diop Y MB, Séne M. 2008. Usage des pesticides et risques sanitaires sur la production horticole de la zone des Niayes au Sénégal. Rev. Sen. Rech. Agri. Agroalim, 03 (2008): 19-26.

Cissé I, Fall ST, Badiane M, Diop Y, Diouf A. 2006. Horticulture et usage des pesticides dans la zone des Niayes au Sénégal, ISRA/LNERV/EISMV/LACT/Faculté de Médecine Pharmacie, UCAD, $8: 14$ p.

Commission du Codex Alimentarius. 2004. Directives Générales sur l'échantillonnage, $\mathrm{CAC} / \mathrm{GL} \quad 50$ 2004CAC/GL 50-2004. 77p.

Deguine JP, Ferron P, Russell D. 2008. Protection des Cultures de l'Agrochimie à l'Agroécologie. Editions Quae, 20 rue des Grands-Augustins : Paris 6 $6^{\mathrm{e}} ; 187 \mathrm{p}$.

Dem SB, Cobb JM, Mullins DE. 2007. Pesticide residues in soil and water from four cotton growing areas of Mali, West Africa. Journal of Agricultural, Food and Environmental, 1: 134-152.

Diop A. 2013. Diagnostic des pratiques d'utilisation et quantification des pesticides dans la zone des Niayes de Dakar (Sénégal). Thèse de doctorat de l'Université du Littoral Côte d'Opale, p. 241.

Diop A, Diop YM, Thiare DD, Cazier F, Sarr SO, Kasprowiak A, Landy D, Delattre F. 
2016. Monitoring survey of the use patterns and pesticide residues on vegetables in the Niayes zone, Senegal. Chemosphere, 144 : 1715-1721. DOI : https://doi.org/10.1016/j.chemosphere.20 15.10 .058

FAO. 2004. Guide sur la gestion et la conservation des sols et des éléments nutritifs pour les champs-écoles des agriculteurs. $176 \mathrm{p}$.

Gouda AI, Toko II, Salami SD, Richert M, Scippo ML, Kestemont P, Schiffers B. 2018. Pratiques phytosanitaires et niveau d'exposition aux pesticides des producteurs de coton du nord du Bénin. Cah. Agric., 27: 65002 DOI : https://doi.org/10.1051/cagri/2018038

ISO 10381. 2005. Qualité du sol Échantillonnage - Partie 5: Lignes directrices pour la procédure d'investigation des sols pollués en sites urbains et industriels. $44 \mathrm{p}$.

ISO 10382. 2002. Qualité du sol — Dosage des pesticides organochlorés et des biphényles polychlorés - Méthode par chromatographie en phase gazeuse avec détection par capture d'électrons. 23p.

ISO 5667-6. 2014. Qualité de l'eau -Échantillonnage -- Partie 6: Lignes directrices pour l'échantillonnage des rivières et des cours d'eau. $28 \mathrm{p}$.

ISO 6468. 1996. Qualité de l'eau -- Dosage de certains insecticides organochlorés, des polychlorobiphényles et des chlorobenzènes -- Méthode par chromatographie en phase gazeuse après extraction liquide-liquide. $25 \mathrm{p}$.

ISO/IEC 17025. 2005. Exigences générales concernant la compétence des laboratoires d'étalonnages et d'essais. 38p.

Kanda M, Wala K, Batawila K, DjaneyeBoundjou G, Ahanchede A, Akpagana K. 2009. Le maraîchage périurbain à Lomé : pratiques culturales, risques sanitaires et dynamiques spatiales. Cah. Agric., 18(4): 356-363. DOI: 10.1684/agr.2009.0319 .

Labou B, Bordat D, Brevault T, Diarra K. 2016. Importance de la "Teigne du chou" dans les Niayes au Sénégal : interrelations avec la température et les cultivars utilisés. Int. J. Biol. Chem. Sci., 10(2): 706-721. DOI : http://dx.doi.org/10.4314/ijbcs.v10i2.21

Labou B, Bordat D, Brévault T, Diarra K. 2017. Spatiotemporal distribution and impact of diamondback moth parasitoids in the Dakar Niayes in Senegal. Int. J. Biol. Chem. Sci., 11(3): 1288-1298. DOI : https://dx.doi.org/10.4314/ijbcs.v11i3.28

Mambe-Ani P, Ouattara KN, Elleingand FE, KADJO V. 2019. Assessment of the impact of pesticide use in urban and periurban agriculture in Abidjan, Côte d'Ivoire. Int. J. Biol. Chem. Sci., 13(6): 2824-2837.

DOI : https://dx.doi.org/10.4314/ijbcs.v13i6.32

NF-EN 15662. 2018. Aliments d'origine végétale - Multiméthode de détermination des résidus de pesticides par analyse $\mathrm{CG}$ et CL après extraction/partition avec de l'acétonitrile et purification par SPE dispersive - Méthode modulaire QuEChERS. 96p. DOI : https://www.boutique.afnor.org/norme/nf -en-15662

Ngom S, Manga A, Diop M, Thiam MB, Rousseau J, Cisse I, Traore S. 2013. Étude de l'évolution des résidus de pesticides dans les produits horticoles de grande consommation au Sénégal. Rev. Ivoir. Sci. Technol., 21\&22: $31-44$. URL: https://revist.net/REVIST_21\&22/REVIS T_21\&22_3

Ngom S, Seydou T, Thiam MB, Anasthasie M. 2012. Contamination des produits agricoles et de la nappe phréatique par les pesticides dans la zone des Niayes au Sénégal. Rev. Sci. Technol., 25: 119-130. URL: https://www.ajol.info/index.php/srst/article/v iew/117245

OMS. 2017. Directives de Qualité pour l'Eau de Boisson : 4e édn intégrant le premier additif [Guidelines for drinking-water quality: 4th edn incorporating first addendum]. 564p.

Régnault RC. 2005. Enjeux Phytosanitaires pour l'Agriculture et l'Environnement. 
Editions Tec \& Doc; p. 1010.

Roche PA, Billen G, Bravard JP, Décamps H, Pennequin D, Vindimian E, Wasson JG. 2005. Les enjeux de recherche liés à la directive-cadre européenne sur l'eau. Comptes Rendus Geoscience, 337(1-2): 243-267.

DOI : https://doi.org/10.1016/j.crte.2004.10.012

Samake F, Babana A H, Yaro F K, Cisse D, Traore I, Kante F, Kone S, Diallo A, Toure H, Toure O. 2011. Risques sanitaires liés à la consommation des produits maraîchers cultivés dans la zone urbaine et périurbaine de Bamako. Mali Santé Publique, 1(1): 27-31.

Soro G, Wahabi SA, Adjiri OA, Soro N. 2019. Risques sanitaires et environnementaux liés à l'usage des produits phytosanitaires dans l'horticulture à Azaguié (Sud Côte d'Ivoire). Journal of Applied Biosciences, 138: $\quad$ 14072-14081. DOI: https://dx.doi.org/10.4314/jab.v138i1.7

Soumaré, CH. 2008. Arrêté primatoral N ${ }^{\circ} 09415$ $\mathrm{du} 16 / 11 / 2008$ portant interdiction d'importation, de production et d'utilisation des pesticides et produits chimiques visés par la convention de Stockholm sur les polluants organiques persistants (POPs). Journal Officiel, République du Sénégal, Dakar. URL: http://www.jo.gouv.sn/spip.php?article73 24

Sow G, Diarra K, Arvanitakis L, Bordat D. 2013. The relationship between the diamondback moth, climatic factors, cabbage crops and natural enemies in a tropical area. Folia Horticulturae, 25(1): 3-12. DOI : https://doi.org/10.2478/fhort2013-0001

Sow G, Diarra K. 2013. Laboratory evaluation of toxicity of Bacillus thuringiensis, neem oil and methamidophos against Plutella xylostella L. (Lepidoptera: Plutellidae) larvae. International Journal of Biological and Chemical Sciences, 7(4): 1524-1533. DOI: http://dx.doi.org/10.4314/ijbcs.v7i4.9.

Sow M, Marone M, Ndiaye S, Mullie WC. 2004. Étude Socio-économique de l'utilisation des pesticides au Sénégal. République du Sénégal, Ministère de l'Agriculture, Ministère de l'Environnement, de la Protection de la Nature, des Bassins. 146p.

Tendeng E, Labou B, DJIBA S, Diarra K. 2017. Actualisation de l'entomofaune des cultures maraîchères en Basse Casamance (Sénégal). Int. J. Biol. Chem. Sci., 11(3): 1021-1028.

DOI: https://dx.doi.org/10.4314/ijbcs.v11i3.7

Traoré SK, Koné M, Dembele A, Lafrance P, Mazelliert P, Houenou P. 2006. Contamination de l'eau souterraine par les pesticides en régions agricoles en Côte d'Ivoire (Centre, Sud et Sud-Ouest). Journal Africain Des Sciences de l'Environnement, 1: 1-9. 